\title{
IMAGING NEGATIVE PARATHYROID ADENOMA
}

\author{
MD. ABDUL KADER ${ }^{1}$, MD ABDUL KALAM AZAD ${ }^{2}$, MD FERDOUS UR RAHAMAN ${ }^{3}$, ABED HUSSAIN \\ $\mathrm{KHAN}^{3}$, MA JALIL CHOWDHURY ${ }^{4}$, MD. MOHIBUL AZIZ ${ }^{5}$, ENAYET KARIM ${ }^{6}$
}

\begin{abstract}
Primary hyperparathyroidism typically presents with skeletal and renal manifestations. In most (80-85\%) cases, the cause is a single parathyroid adenoma. We present a case of 36-year-old lady of primary hyperparathyroidism presented with multiple fractures, nephrolithiasis, and nephrocalcinosis. All of the imaging techniques to localize the cause were negative. She underwent surgical neck exploration with detection of left parathyroid adenoma. Her post-operative period was uneventful with significant clinical and biochemical improvement.
\end{abstract}

Keyword: Primary hyperparathyroidism, hyperparathyroidism, hypercalcemia, parathyroid imaging.

\section{Introduction}

Primary hyperparathyroidism (PHPT) is an endocrine disorder, characterized by excessive secretion of parathyroid hormone (PTH) from one or more parathyroid glands ${ }^{1}$. The clinical spectrum of primary hyperparathyroidism has undergone a striking change in developed countries, with the predominant form being asymptomatic hyperparathyroidism, detected on routine biochemical screening for other causes. ${ }^{2-6}$ However, the disease continues to present with classic florid skeletal and renal manifestations in subcontinent. ${ }^{7-10}$ Primary hyperparathyroidism is increasingly being recognized because of the widespread use of general multiphasic screening. Several reports have stated that in experienced hands, some $90 \%-95 \%$ of parathyroid adenomas will be localized during an initial neck exploration. Here we report a 36-yearold lady of PHPT presented with multiple fractures, nephrolithiasis, and nephrocalcinosis. All of the imaging techniques to localize the cause were negative. She underwent surgical neck exploration with detection of left parathyroid adenoma. Her postoperative period was uneventful with significant clinical and biochemical improvement.

\section{Case Report}

A 36-years-old lady was incidentally diagnosed as case of nephrolithiasis, nephrocalcinosis and right ureteric calculus which was confirmed by intravenous urography (IVU) on $17^{\text {th }}$ September during her hospital stay for treatment of fibroid uterus. Ureterolithotomy was done and chemical analysis of the stone revealed oxalate stone. Subsequently after 2-3 months she started passing of stone per urethra and she also complaints of progressively increasing generalized bodyache associated with bone pain. For these complaints she was investigated and diagnosed as a case of primary hyperparathyroidism based on raised PTH, raised calcium and decreased phosphate. Further investigations including $\mathrm{CT}, \mathrm{MRI}$ of the neck and parathyroid scan were unremarkable. However the patient deteriorated with multiple low-trauma fracture of the right clavicle, vertebral fracture and shaft of the right femur on January 2008. With this problem, she got admitted in Bangabandhu Sheikh Mujib Medical University (BSMMU). She was again investigated to confirm PHPT and localize the lesion.

During her stay at BSMMU, her serum calcium was $14 \mathrm{mg} / \mathrm{dl}$ (ref:8.5-10.5mg/dl), parathyroid hormone $1611 \mathrm{pg} / \mathrm{ml}$ (ref: 9-80 pg/ml), phosphate $2.4 \mathrm{mg} / \mathrm{dl}$ (2.5$4.5 \mathrm{mg} / \mathrm{dl})$; her renal function was mildly impaired serum creatinine $(1.56 \mathrm{mg} / \mathrm{dl})$.

1. Assistant Professor, Department of Medicine, Bangabandhu Sheikh Mujib Medical University

2. Associate Professor, Department of Medicine, BSMMU

3. Medical Officer, Department of Medicine, BSMMU

4. Professor, Department of Medicine, BSMMU

5. Associate Professor, Department of Surgery, BSMMU

6. Associate Professor, Department of Radiology \& Imaging, BSMMU

Correspondence: Md. Abdul Kalam Azad, Associate Professor of Medicine, Bangabandhu Sheikh Mujib Medical University, E-mail: azadbsmmu@yahoo.com 


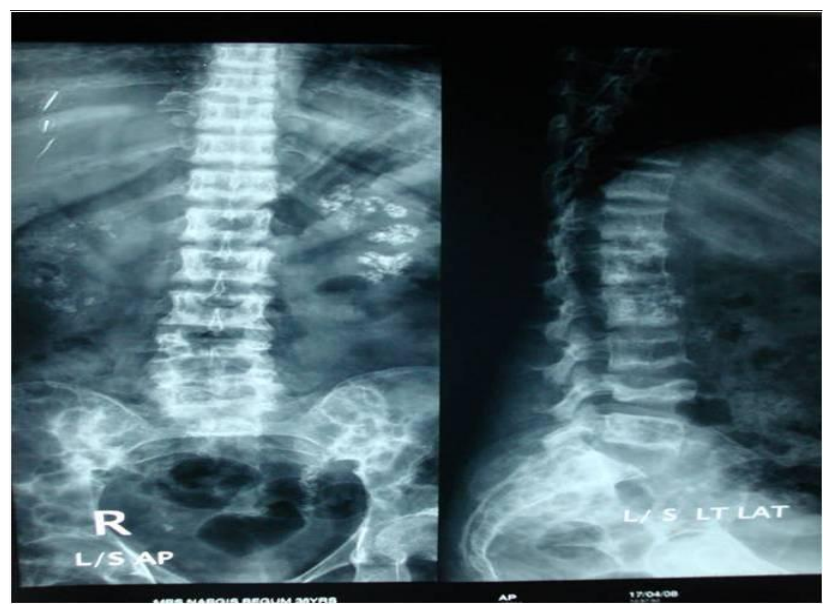

Fig.-1: Nephrocalcinosis with multiple osteolytic lesions in vertebrae and hip bones with wedge fracture of 4 th Lumber vertebrae

Radiological survey revealed gross osteopenia, punched out osteolytic lesion and fractures (Figures $1 \& 2$ ). CT scan, MRI of the neck and parathyroid scintigraphy were unremarkable.

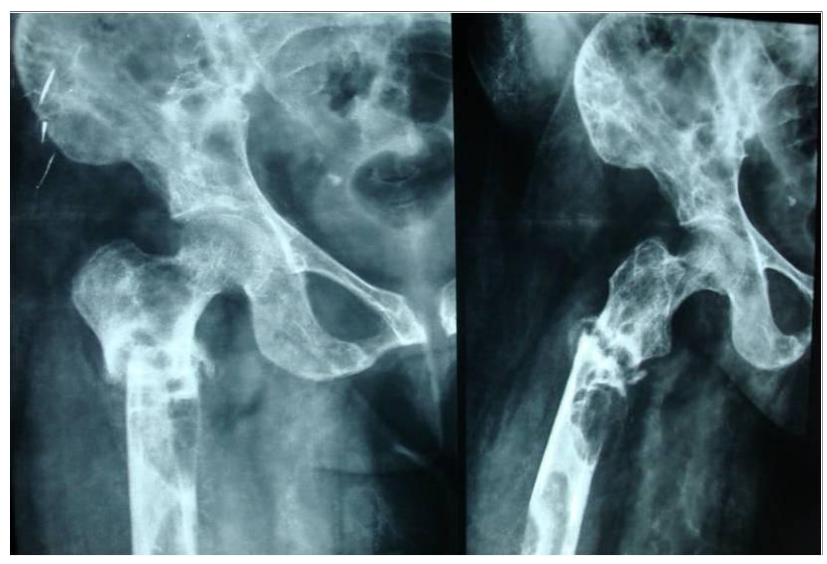

Fig.-2: Multiple osteolytic lesions with fracture in shaft of right femur

Considering the clinical diagnosis of primary hyperparathyroidism, she was referred to endocrine surgeon. On surgical exploration an enlarged left superior parathyroid gland measuring $4 \mathrm{~mm} \times 0.5 \mathrm{~mm}$ was identified and was removed. Histopathological examination of the excised gland revealed a benign tumor composed of polygonal cells arranged diffusely and in follicular pattern. The individual cells show abundant eosinophilic cytoplasm and small round nuclei suggestive of parathyroid adenoma. The post operative period was uneventful and her serum calcium levels returned to normal.

\section{Discussion:}

Primary Hyperparathyroidism (PHPT) is present in about $1 \%$ of the adult population. Incidence increases to $2 \%$ or higher after age 55 years and is 2 to 3 times more common in women than in men. In India the average age at presentation is $30-40$ years.

In most (80-85\%) cases, the cause is a single parathyroid adenoma. In the remainder, hyperplasia of the parathyroid gland (10\%), a double parathyroid adenoma $(4 \%)$ or parathyroid carcinoma $(<1 \%)$ is the cause.

The clinical features of PHPT are mainly due to the direct and indirect effects of excess PTH on the skeleton, kidneys, and intestine and normally include bone resorption of calcium and phosphorus, enhanced intestinal absorption of calcium, renal tubular reabsorption of calcium, and hypercalciuria, resulting hypercalcaemia and hypophosphataemia. Most patients with PHPT are asymptomatic. Symptomatic patients may present with history of renal calculi, bone pain, pathologic fractures, bone shaft tumors, proximal muscle weakness (especially of the lower extremities), or nonspecific symptoms such as depression, lethargy, and vague aches and pains.

Immunoassay of the intact PTH molecule is the preferred method of measurement. This diagnosis should be suspected in symptomatic patients (typically with renal calculi, renal dysfunction, osteopenia, or osteoporosis) who have minimally increased PTH levels and high-normal serum calcium levels (normocalcemic PHPT) or intermittently increased serum calcium levels (intermittent PHPT). Dual-energy x-ray absorptiometry (DXA) scan is necessary for screening and monitoring of osteopenia and osteoporosis.

Parathyroid imaging has minimum role in the diagnosis of PHPT; the thalium-technetium subtraction method gave a sensitivity of only $73 \%$, which compares favorably with the sensitivities of $76 \%$ for $\mathrm{CT}$ scanning, $55 \%$ for high resolution ultrasonography and 50-93\% for magnetic resonance imaging. ${ }^{11,12}$ Combination of CT scanning and radionuclide imaging achieved a sensitivity of $90 \%$ and the combination of radionuclide imaging and ultrasound, $85 \%{ }^{13}$

Parathyroidectomy is indicated in patients who meet some of the following criteria: (1) age $<50$ years; (2) serum calcium concentrations $>1.0 \mathrm{mg} / \mathrm{dl}$ above the 
upper limit of normal; (3) $24 \mathrm{~h}$ urinary calcium $>400$ mg; (4) creatinine clearance reduced by $30 \%$; (5) reduced bone mineral density (BMD; T score $<-2.5)$ at any site. Open parathyroidectomy is the treatment of choice in PHP.

Several drugs have been used in the medical management of PHP. These can be categorized into two groups: (1) agents that act directly on PTH secretion at parathyroid cell level i.e. Calcimimetics, in particular cinacalcet; (2) those that act on PTH target organs, mainly at bone level i.e. antiresorptive drugs, such as bisphosphonates, estrogens and selective estrogen-receptor modulators (SERMs).

\section{Conclusion}

High clinical suspicion with biochemical support is essential for early diagnosis and prevention of complications of primary hyperparathyroidism. Imaging study may not help in the localization of the adenoma. Neck exploration by an expert surgeon can identify a parathyroid adenoma per-operatively. Removal of the pathological parathyroid tissue is the main therapeutic target in the management of PHP. Surgical treatment is considered the treatment of choice because it is associated with a high success rate with minimal surgical complications.

\section{References}

1. Bilezikian JP, Brandi ML, Rubin M. Primary hyperparathyroidism: new concepts in clinical, densitometric and biochemical features. J Intern Med 2005; 257: 6-17.

2. Wermers RA, Khosla S, Atkinson EJ. The rise and fall of primary hyperparathyroidism: A populationbased study in Rochester, Minnesota, 1965-1992. Ann Intern Med 1997; 126: 433-40.

3. Heath H, Hodgson SF, Kennedy MA. Primary hyperparathyroidism. Incidence, morbidity and potential economic impact in a community. N Eng $\mathrm{J}$ Med 1980; 302: 189-93.

4. Cope O. The study of hyperparathyroidism at the Massachusetts General Hospital. N Eng J Med 1966; 274: 1174-82.

5. Mallette LE, Bilezikian JP, Heath DA. Primary hyperparathyroidism: clinical and biochemical features. Medicine (Baltimore) 1974; 53: 127-46.

6. Bilezikian JP, Silverberg SJ, Shane E. Characterization and evaluation of asymptomatic primary hyperparathyroidism. J Bone Miner Res 1991; 6 :(Suppl 2): S 85-89.

7. Kapur MM, Agarwal MS, Gupta A. Clinical \& biochemical features of primary hyperparathyroidism. Indian J Med Res 1985; 81: 607-12.

8. Harinarayan CV, Gupta N, Kochupillai N. Vitamin D status in primary hyperparathyroidism in India. Clin Endocrinol (Oxf) 1995; 43: 351-58.

9. Mishra SK, Agarwal G, Kar DK. Unique clinical characteristics of primary hyperparathyroidism in India. Br J Surg 2001; 88: 708-14.

10. Hansali A, Masoodi SR, Reddy KS. Primary hyperparathyroidism in North India: a description of 52 cases. Ann Saudi Med 2005; 25: 29-35.

11. Miller DL, DoppmanJL, Krydy AG, et al. Localization of parathyroid adenomas in patient who have undergone surgery. Part I. Non-invasive imaging methods. Radiol 1987; 162:133-37.

12. Young AE, Gaunt JI, Croft DN, et al. Location of parathyroid adenomas by thalium ${ }^{201}$ and technetium $^{99 \mathrm{~m}}$ subtraction scanning. Br Med J 1983; 286:1384-6

13. StarkDD, Gooding GAW, Moss AA, et al. Parathyroid imaging: comparison of high resolution CT and high resolution sonography. Am J Radiol 1983; 141:63338. 